\title{
Quanto è "complessa" la crescita? Un'analisi panel in 27 paesi Europei
}

\author{
Angelo Castaldo*, Andrea Billi**, Gaia Orzi ${ }^{* * *}$
}

\section{Sommario}

Ricevuto 02/07/2020 - Accettato 17/09/2020

Il presente lavoro mira a costruire un ponte tra la letteratura sulla complessità economica e i modelli teorici della crescita endogena. I modelli tradizionali di crescita, infatti, sembrano sottostimare l'impatto generato dalle interazioni sistemiche dei diversi fattori di crescita. Per colmare tale gap, si adatta il modello di Mankiw et al. (1992) al fine di poter includere una misura di complessità economica (i.e., Economic Complexity Index - ECI). L'analisi empirica è riferita ad un panel (20072018) di 27 paesi dell'area europea. Con il ricorso ad un approccio tradizionale (panel a effetti fissi e random), dinamico auto-regressivo (system-GMM) e semi-parametrico ad effetti fissi, l'analisi condotta evidenzia che il livello di complessità dei sistemi economici incide significativamente sui livelli di crescita economica e che la relazione funzionale è di tipo non lineare.

Parole chiavi: Complessità economica; Modelli di crescita endogena; Indice di complessità economica; GMM; modello semi-parametrico a effetti fissi.

\section{Abstract \\ How "complex" is growth? A panel analysis in 27 European area countries}

This paper aims to build a bridge between the economic complexity and endogenous growth stream of works. Traditional growth empirical and theoretical literature tend to underestimate the relevance of the effects exerted by the systematic interactions

${ }^{*}$ Ricercatore e Professore Aggregato in Scienza delle Finanze. Dipartimento di Studi Giuridici ed Economici, Sapienza Università di Roma, angelo.castaldo@uniroma1.it Corresponding author.

** Ricercatore e Professore Aggregato in Economia Politica. Dipartimento di Studi Giuridici ed Economici, Sapienza Università di Roma, andrea.billi@uniroma1.it

*** Dottoranda in Studi Politici - Teorie dei Processi Socio-Culturali, Politici e della Cooperazione Internazionale, Sapienza Università di Roma. gaia.orzi@uniroma1.it

Corporate Governance and Research \& Development Studies, n. 1-2020

(ISSN 2704-8462-ISSNe 2723-9098, DOI: 10.3280/cgrds1-2020oa10104) 
among the growth factors. In this vein, this work adapts the Mankiw et al. (1992) model in order to consider, in the technological component of the growth function, a measure of the country's system economic complexity. The empirical analysis refers to a panel of 27 European area countries for 12 years (2007-2018). Implementing both traditional panel estimation approach (fixed and random effects model) and dynamic generalized method of moments (system-GMM) approach, the findings obtained reveal that the economic complexity index (ECI) represents a primary determinant of actual growth path.

Keywords: Growth theory, Complexity economics; Economic complexity index; GMM; semi-parametric fixed effects model.

\section{Introduzione}

La comprensione del modo in cui i sistemi economici crescono ed evolvono è da sempre una questione centrale nel dibattito tra gli economisti. Accanto ai modelli legati alla teoria della crescita endogena, la scuola strutturalista offre una prospettiva di analisi complementare ed arricchente. I modelli tradizionali di crescita endogena, infatti, pur dando rilevanza alle componenti endogene dello sviluppo economico, sembrano trascurare l'impatto generato dalle interazioni complesse dei diversi fattori di crescita.

Con un approccio prevalentemente empirico, il nostro lavoro mira a verificare se l'inclusione di indicatori di complessità economica all'interno dei modelli di crescita endogena contribuisce a migliorare lo studio della dinamica di crescita dell'economia. L'ipotesi di ricerca che testiamo è che più elevato è il livello di complessità di un sistema economico, più sostenuta è la sua capacità di crescita. In questa prospettiva, come misura di complessità utilizziamo l'Economic Complexity Index - ECI (Hidalgo e Hausmann, 2009; Hausmann et al., 2011; Hausmann et al., 2013; Hidalgo, 2015). L'ECI utilizza i dati sul commercio internazionale e viene interpretato come misura della quantità di knowledge presente nella struttura produttiva di un paese, andando dunque oltre alla semplice accumulazione dei fattori di produzione come il capitale fisico o umano. Le economie complesse sono quelle che riescono a rendere produttive grandi quantità di knowledge attraverso reti di imprese e persone e a dar vita a prodotti diversificati e sofisticati; la gamma dei beni che un paese può produrre, pertanto, rappresenta una misurazione indiretta delle capabilities e del posizionamento competitivo dei diversi sistemi economici.

Attraverso un'analisi panel (2007-2018) in 27 paesi dell'area europea stimiamo il modello di Mankiw et al. (1992) sia con un approccio a effetti fissi e random che auto-regressivo dinamico (GMM), includendo l'indicatore di 
complessità economica (ECI) all'interno della componente tecnologica. Al fine di comprendere il legame funzionale esistente tra complessità e crescita economica, inoltre, stimiamo un modello semi-parametrico ad effetti fissi (Baltagi e Li, 2002) includendo l'ECI nella componente non parametrica.

I risultati ottenuti evidenziano, ceteris paribus, che il livello di complessità dei diversi sistemi economici incide fortemente sui livelli di crescita economica e che la relazione funzionale è non lineare. In particolare, al crescere del livello di complessità economica il Pil reale pro capite cresce ma a tassi decrescenti.

Il lavoro è strutturato come segue. Nel secondo paragrafo mettiamo a sistema la letteratura relativa ai cambiamenti strutturali dei sistemi economici, ai modelli di crescita endogena e alle misure di complessità. Nel terzo paragrafo presentiamo il nostro dataset e la nostra strategia di stima. Successivamente, nel quarto paragrafo presentiamo i principali risultati dell'analisi empirica. Infine, il quinto paragrafo traccia le principali conclusioni.

\section{Complexity e crescita economica}

Il presente lavoro si propone di creare un collegamento tra la recente letteratura sulla complessità economica e i modelli teorici della crescita endogena con l'obiettivo di verificare la possibilità di arricchire le prospettive di analisi dell'andamento del Pil.

Rosenstein-Rodan (1943), pietra miliare dell'approccio iniziale allo strutturalismo, pone particolare enfasi sul potere dell'industrializzazione sul sistema economico. Su questa scia, numerosi lavori affrontano l'analisi dello sviluppo e della crescita focalizzandosi sul ruolo economico della trasformazione strutturale (Prebisch, 1950, 1959; Singer, 1950; Nurkse, 1953; Lewis, 1954; Myrdal, 1957; Hirschman, 1958; Rostow, 1959; Chenery, 1960; Furtado, 1964; Kuznets, 1966; Kaldor, 1967). Gli elementi del sistema economico, interdipendenti tra loro, formano un sistema complesso che include delle proprietà che rendono insufficiente un' analisi rivolta a singoli elementi. Le relazioni che ne sono alla base, seppure non direttamente osservabili, determinano fenomeni economici osservabili. Per Hirschman (1958) lo sviluppo economico è connesso non tanto alla individuazione di combinazioni ottimali di risorse e fattori di produzione, quanto ad una questione di coordinamento di risorse e capacità nascoste $\mathrm{o}$ mancanti. I drivers a capo di questa azione di coordinamento sono per Hirschman le connessioni (linkages) di produzione, che generano investimenti e diversificazione produttiva. Negli stessi anni Solow (1956), partendo dai lavori di Harrod e Domar (Harrod, 
1939; Domar, 1946), elabora il "modello neoclassico di crescita" dimostrando che il risparmio (l'accumulazione del capitale) e la crescita della popolazione (la forza lavoro) contribuiscono solo in parte alla crescita del sistema economico. La quota restante è da imputare al progresso tecnologico, supposto esogeno. I punti lasciati aperti da Solow sono alla base della nascita e affermazione della "nuova teoria della crescita" o "teoria della crescita endogena". Il motore della crescita, pertanto, diviene endogeno (Romer, 1986, 1990, 1994; Lucas, 1988; Barro, 1990; Grossman e Helpman, 1991; Aghion e Howitt, 1992, 1998). Con Romer (1986) la crescita è ricondotta all'esternalità positiva prodotta dall'esperienza acquisita (learning by doing) - concetto questo del quale fu pioniere Arrow (1962). Lucas (1988) introduce nel modello di crescita economica il capitale umano e ne evidenzia la capacità di produrre esternalità positive, rendendolo fattore rilevante dello sviluppo economico. Con il termine capitale umano, Lucas (1988) identifica la capacità e le abilità (skills) dell'individuo, sulla scia di Schultz (1963) e Becker (1964). Il modello di Solow (1956) viene esteso successivamente con il lavoro di Mankiw et al. (1992), il quale include l'accumulazione del capitale umano accanto a quella del capitale fisico.

In termini generali, dunque, emerge la tendenza a riconoscere che la crescita economica sia fortemente influenzata dalle attività legate alla ricerca $\mathrm{e}$ sviluppo e dalla qualità del capitale umano. Se da un lato vengono riconosciute le componenti endogene dello sviluppo economico e il loro relativo apporto alla crescita, dall'altro la letteratura sembra sottostimare le interazioni tra i fattori e l'apporto che queste interazioni determinano alla crescita stessa.

Al contrario, l'approccio della Complexity Economics (Arthur, 1999; Beinhocker, 2006; Mitchell, 2009) studia le proprietà delle interazioni tra gli elementi eterogenei presenti all'interno dei sistemi complessi.

In tempi più recenti, le misure di "complessità economica" hanno ampliato la nostra capacità di quantificare la struttura produttiva di un paese $\mathrm{e}$ il ruolo macroeconomico delle trasformazioni strutturali.

Rifacendosi apertamente alle idee di Hirschman (1958), Hidalgo e Hausmann (2009) introducono il così detto Method of Reflections per caratterizzare la struttura delle reti bipartite, ossia dei dati che connettono i paesi ai beni che esportano. Le variabili così prodotte vengono interpretate come indicatori di complessità economica, ossia di varietà di capabilities presenti in un paese e delle loro interazioni. Gli autori dimostrano che gli indicatori ottenuti con tale approccio sono correlati positivamente al reddito pro-capite dei paesi. Gli indicatori elaborati, infatti, sono predittivi della crescita futura: i paesi tendono al livello di reddito associato alla loro complessità.

Sulla scia del lavoro Hidalgo e Hausmann (2009), Hausmann et al. (2011) 
presentano la formulazione dell'Economic Complexity Index (ECI). L'ECI offre una misura della complessità di un paese, ossia della quantità di conoscenze - di capabilities - incorporate nella sua struttura produttiva.

L'idea alla base dell'ECI è che se un paese esporta un certo bene vuol dire che quel paese possiede le competenze e conoscenze necessarie a produrre quel bene. I beni vengono visti, quindi, come veicoli della conoscenza necessaria a produrli. Da qui emergono due principali implicazioni (Hidalgo e Hausmann, 2009; Hausmann et al., 2011; Hausmann et al., 2013; Hidalgo, 2015):

1) i paesi che possiedono maggiore conoscenza hanno la possibilità di produrre un più ampio set di beni. Perciò l'ammontare di conoscenza presente in un paese è riflesso nella sua varietà produttiva (i.e., economies of diversification);

2) i beni che richiedono un ampio volume di conoscenza possono essere prodotti solo nei paesi dotati delle competenze necessarie. Perciò il numero di paesi che producono un determinato bene rivela informazioni sul volume di conoscenza richiesto per il processo produttivo di quel bene.

Hidalgo e Hausmann (2009), per strutturare indicatori idonei a cogliere la conoscenza posseduta da un paese, propongono due misure: la diversity e l'ubiquity. La diversity di un paese è misurata dal numero di prodotti al quale un paese è connesso, ossia il numero di prodotti esportati da quel paese con vantaggio comparato rivelato (Revealed Comparative Advantage- $R C A)^{1}$, ed è una proxy della varietà di capabilities disponibile in un paese. La ubiquity di un prodotto, invece, viene espressa dal numero di paesi al quale un prodotto è connesso, ossia dal numero di paesi che esportano quel prodotto con RCA; anch'essa è una proxy della varietà di capabilities richieste da un prodotto.

Secondo tale filone di letteratura (Hidalgo e Hausmann, 2009; Hausmann et al., 2011; Hausmann et al., 2013), la marcata disuguaglianza di redditi tra paesi è espressione dei significativi differenziali di conoscenza (prevalentemente "tacita") tra i diversi sistemiei economici nazionali. In tale prospettiva (Hidalgo, 2015), le misure di complessità e di diversity vengono interpretate anche come proxy dell'endowment di capitale sociale e della qualità delle istituzioni. Hidalgo (2015) e Fukuyama (1996) ritengono, infatti, che la capacità di un paese di produrre beni sofisticati dipende anche dalla capacità delle persone di creare reti sociali e professionali che consentano di coordinare e condividere la conoscenza ${ }^{2}$. Per questo motivo, i prodotti industriali complessi, rispetto ai prodotti più semplici, tendono a richiedere un più ampio volume di "conoscenza tacita" e di "conoscenza distribuita". Le economie complesse sono quelle in grado di dar vita a reti (ad es. networks) di

\footnotetext{
${ }^{1}$ Balassa (1965).

${ }^{2}$ Hidalgo (2015) e Fukuyama (1996).
} 
individui e organizzazioni capaci di assemblare e rendere produttive le diverse competenze esistenti.

L'obiettivo del presente lavoro è di includere l'indicatore di complessità economica elaborato da tale filone di letteratura (ECI) all'interno della componente tecnologica del modello di crescita economica endogena proposto da Mankiw et al. (1992). Riteniamo che la tecnologia contenga delle componenti sistemiche che non vengono totalmente internalizzate nelle stime empiriche dei modelli di crescita. La spesa in ricerca e sviluppo, d'altronde, pur rappresentando un fattore rilevante del livello di competitività dei paesi e dei trend di crescita economica, non può cogliere tutti gli elementi rivelatori del grado di complessità dei sistemi innovativi. Mancano indicatori sintomatici delle interazioni tra i fattori produttivi, ovvero, in grado di considerare l'apporto offerto dall'interazione alla crescita. In quest'ottica, al fine di cogliere la rilevanza delle componenti sistemiche all'interno dell'analisi empirica dei trend di crescita economica, utilizziamo l'indicatore dell'ECI come principale variabile strategica. In termini più sintetici, il nostro quesito di ricerca mira a verificare se l'ECI riesca o meno a cogliere aspetti che sfuggono alle determinanti classiche dello sviluppo economico e, quindi, se debba trovare spazio nella specificazione dei modelli di stima.

Il nostro lavoro empirico si inserisce nel filone di letteratura che cerca di instaurare una connessione tra i modelli di complessità economica e i modelli di crescita tradizionali. In particolare, Ferrarini e Scaramozzino (2016) elaborano una misura di complessità partendo dal concetto di Product Space di Hausmann e Klinger (2007), utilizzandola nella specificazione di un modello di crescita endogena con accumulazione di capitale umano.

Stojkoski e Kocarev (2017) propongono, nello spirito della teoria della crescita endogena, un modello econometrico per descrivere la relazione di lungo e breve periodo tra complessità economica e crescita economica nelle regioni dell'Europa sud-orientale e centrale.

Sbardella et al. (2018), nell'analizzare le determinanti dell'impatto sulla crescita economica, utilizzano un approccio non parametrico che combina la Economic Fitness (misura delle capabilities di un paese) con alcuni drivers tradizionali della crescita.

Gala et al. (2018) si pongono l'obiettivo di verificare se l'eterogeneità dei livelli di complessità riscontrata nei diversi paesi sia rilevante nel processo di convergenza e divergenza tra paesi ricchi e poveri.

Su questa scia il nostro lavoro si differenzia dai precedenti per la scelta delle variabili impiegate e la metodologia utilizzata. In particolare, l'analisi empirica poggia sull'approccio panel dinamico auto-regressivo systemGMM e l'approccio semi-parametrico ad effetti fissi (Baltagi e Li, 2002). 


\section{Dati e strategia di stima}

\section{a) Dati}

Il nostro panel è composto da 27 paesi (Austria, Bulgaria, Cipro, Danimarca, Estonia, Finlandia, Francia, Germania, Grecia, Irlanda, Italia, Lettonia, Lituania, Norvegia, Paesi Bassi, Polonia, Portogallo, Repubblica Ceca, Regno Unito, Romania, Slovacchia, Slovenia, Spagna, Svezia, Svizzera, Turchia, Ungheria) per 12 anni (2007-2018). La scelta tra i paesi dell'area dell'Europa geografica è condizionata dall'esigenza di garantire, per ogni variabile trattata, serie storiche complete per ognuno dei 12 anni presi in considerazione.

\section{b) Variabili di interesse}

La tabella 1 riporta le descrizioni delle variabili utilizzate. La fonte dei dati è Eurostat eccetto per la popolazione in età lavorativa (fonte OECD) e l'Economic Complexity Index (fonte Observatory of Economic Complexity Index).

Tab. 1 - Descrizione delle variabili

\begin{tabular}{|c|c|c|}
\hline Variabile & Descrizione & Fonte \\
\hline Pil pro capite & $\begin{array}{l}\text { Prodotto Interno Lordo reale pro capite } \\
\text { (euro) in parità di potere di acquisto. }\end{array}$ & Eurostat \\
\hline Investimenti fissi lordi & $\begin{array}{l}\text { Acquisizioni, al netto delle cessioni, di ca- } \\
\text { pitale fisso effettuate dai produttori resi- } \\
\text { denti a cui si aggiungono gli incrementi di } \\
\text { valore dei beni materiali non prodotti (per- } \\
\text { centuale del Pil). }\end{array}$ & Eurostat \\
\hline Occupazione & $\begin{array}{l}\text { Percentuale di persone occupate rispetto al } \\
\text { totale della popolazione. }\end{array}$ & Eurostat \\
\hline $\begin{array}{l}\text { Popolazione in età lavora- } \\
\text { tiva }\end{array}$ & $\begin{array}{l}\text { Percentuale della popolazione tra } \mathrm{i} 15 \text { e } \mathrm{i} \\
64 \text { anni di età rispetto al totale della popo- } \\
\text { lazione. }\end{array}$ & OECD \\
\hline $\begin{array}{l}\text { Economic Complexity In- } \\
\text { dex (ECI) }\end{array}$ & $\begin{array}{l}\text { Misura della complessità di un paese, ossia } \\
\text { della quantità di conoscenza incorporata } \\
\text { nella sua struttura produttiva. È ottenuta } \\
\text { combinando l'informazione sulla diversity } \\
\text { di un paese (il numero di prodotti esportati) } \\
\text { e l'informazione sulla ubiquity dei suoi } \\
\text { prodotti (il numero di paesi che esportano } \\
\text { quel prodotto). Tali indicatori sono spiegati } \\
\text { a pag. } 6 \text { (eq. } 5 \text { e } 6 \text { ). }\end{array}$ & $\begin{array}{l}\text { Observatory } \\
\text { of Economic } \\
\text { Complexity } \\
\text { (MIT) }\end{array}$ \\
\hline Spesa pubblica in $R \& S$ & $\begin{array}{l}\text { Spesa pubblica in ricerca e sviluppo (in } \\
\text { percentuale del Pil). }\end{array}$ & Eurostat \\
\hline Spesa privata in $R \& S$ & $\begin{array}{l}\text { Spesa privata in ricerca e sviluppo (in per- } \\
\text { centuale del Pil). }\end{array}$ & Eurostat \\
\hline Debito pubblico & $\begin{array}{l}\text { Percentuale di debito pubblico rispetto al } \\
\text { Pil. Espresso in euro. }\end{array}$ & Eurostat \\
\hline
\end{tabular}




\section{c) Statistiche descrittive}

La tabella 2, inoltre, riporta le principali statistiche descrittive delle variabili (media, deviazione standard, valore minimo e massimo).

\begin{tabular}{|c|c|c|c|c|c|}
\hline Variabili & $O b s$ & Mean & Std. Dev. & Min & $\operatorname{Max}$ \\
\hline Pil pro capite & 324 & $25,693.33$ & $15,950.33$ & 4,820 & 69,530 \\
\hline Investimenti fissi lordi & 324 & 21.9 & 4.182 & 11.074 & 37.287 \\
\hline Occupazione & 324 & 70.462 & 7.107 & 47.8 & 82.5 \\
\hline Popolazione in età lavorativa & 324 & 66.73 & 2.048 & 62.138 & 72.411 \\
\hline Economic Complexity Index & 324 & 1.15 & .469 & .228 & 2.17 \\
\hline Spesa pubblica in R\&S & 324 & .628 & .234 & .2 & 1.13 \\
\hline Spesa privata in $R \& S$ & 324 & .983 & .711 & .07 & 2.67 \\
\hline Debito pubblico & 324 & 61.656 & 35.402 & 3.8 & 181.2 \\
\hline
\end{tabular}

Come anticipato, in questo lavoro ci proponiamo di stabilire una connessione tra la letteratura sulla complessità economica e i modelli di crescita. Come misura di complessità viene utilizzato l'Economic Complexity Index (ECI) formulato in Hausmann et al. (2011) e Hausmann et al. (2013). I dati relativi all'ECI sono stati presi dall'Observatory of Economic Complexity (MIT) per il periodo 2007-2018 ${ }^{3}$. Per il nostro panel, in ottica comparativa, si può osservare che Svizzera (2.029), Germania (1.916) e Svezia (1.731) sono i tre paesi che in media hanno i più alti valori di ECI nel periodo considerato. Bulgaria $(0.530)$, Turchia $(0,524)$ e Grecia $(0,266)$ sono invece in fondo alla classifica.

I dati sul commercio internazionale vengono interpretati come reti bipartite nelle quali i paesi sono connessi ai prodotti che esportano. Una rete bipartita è un insieme di nodi che può essere partizionato in due sottoinsiemi tali che ciascun link connette solo nodi di differenti insiemi. Matematicamente questa rete è rappresentata da una matrice di adiacenza $M_{c p}$ che è pari a 1 se il paese $c$ è un esportatore significativo del prodotto $p$ ed è pari a 0 altrimenti.

$M_{c p}=\left\{\begin{array}{l}1 \text { se } R C A_{c p} \geq 1 \\ 0 \text { negli altri casi }\end{array}\right.$

Il paese $c$ è considerato un esportatore significativo del prodotto $p$ se il suo vantaggio comparato rivelato (Revealed Comparative Advantage -

\footnotetext{
${ }^{3}$ I dati sul commercio internazionale, indispensabili per la costruzione dell'indicatore di complessità economica, provengono dal BACI International Trade Database, che utilizza dati provenienti dalla United Nations Statistical Division (Comtrade) e segue la classificazione HS - Harmonized System (World Customs Organization) con classificazione a 4 digit.
} 
RCA) è maggiore o uguale al valore soglia 1 (Balassa, 1965). L'indice di vantaggio comparato rivelato è un indicatore costruito a partire dalla composizione settoriale dei flussi commerciali registrati in un dato periodo.

Formalmente, se $X_{c p}$ rappresenta le esportazioni di un paese $c$ del prodotto $p$, l'RCA è espresso dalla seguente formula.

$R C A_{c p}=\frac{X_{c p}}{\sum_{c} X_{c p}} / \frac{\sum_{p} X_{c p}}{\sum_{c, p} X_{c p}}$

I paesi e i prodotti vengono caratterizzati introducendo una famiglia di variabili che cattura la struttura della rete definita da $M_{c p}$. Data la simmetria della rete bipartita, gli autori attribuiscono a questa tecnica il nome di Method of Reflections (Hidalgo e Hausmann, 2009). Il risultato è un set simmetrico di variabili per i due tipi di nodi presenti nella rete (paesi e prodotti). Il $\mathrm{Me}$ thod of Reflections consiste nel calcolare il valore medio delle proprietà di un intorno di un nodo attraverso il valore delle proprietà del nodo precedente, si calcola quindi con un metodo iterativo.

$$
\begin{aligned}
& k_{c, N}=\frac{1}{k_{c, 0}} \sum_{p} M_{c p} \cdot k_{p, N-1} \\
& k_{p, N}=\frac{1}{k_{p, 0}} \sum_{c} M_{c p} \cdot k_{c, N-1}
\end{aligned}
$$

Dove $N$ indica il numero di iterazioni.

Il Method of Reflections è uno strumento che permette di esprimere le proprietà di un nodo di una rete come combinazione delle proprietà dei nodi vicini e nel caso specifico permette di migliorare la comprensione della struttura produttiva dei paesi e della sofisticatezza dei prodotti.

A condizioni iniziali, $\operatorname{con} N=0$, si ha:

$$
\begin{aligned}
& k_{c, 0}=\sum_{p} M_{c p} \\
& k_{p, 0}=\sum_{c} M_{c p}
\end{aligned}
$$

che rappresentano, rispettivamente, i livelli osservati di diversity di un paese (il numero di prodotto esportati da quel paese - il numero di prodotti al quale un paese è connesso) e la ubiquity di un prodotto (il numero di paesi che esportano quel prodotto - il numero di paesi al quale un prodotto è connesso). 
Inserendo la (4) nella (3) si ottiene:

$$
\begin{aligned}
& k_{c, N}=\frac{1}{k_{c, 0}} \sum_{p} M_{c p} \frac{1}{k_{p, 0}} \sum_{c^{\prime}} M_{c^{\prime} p} \cdot k_{c^{\prime}, N-2} \\
& k_{c, N}=\sum_{c^{\prime}} k_{c^{\prime}, N-2} \cdot \sum \frac{M_{c p} M_{c^{\prime} p}}{k_{c, 0} k_{p, 0}}
\end{aligned}
$$

Che viene riscritta come:

$$
k_{c, N}=\sum_{c \prime} \widetilde{M}_{c c} \cdot k_{c^{\prime}, N-2}
$$

Dove:

$$
\widetilde{M}_{c c^{\prime}}=\sum_{p} \frac{M_{c p} M_{c \prime p}}{k_{c, 0} k_{p, 0}}
$$

e $c^{\prime}$ appartiene all'insieme dei paesi considerati.

L'equazione (9) è soddisfatta quando $k_{c, N}=k_{c, N-2}=1$, che rappresenta l'autovettore di $\widehat{M_{C C}}$, il quale è associato all'autovalore maggiore. Dato che l'autovettore è un vettore di soli 1, non è informativo. Si guarda dunque all'autovettore associato al secondo più grande autovalore. Questo è l'autovettore che cattura meglio la varianza del sistema e rappresenta la misura di complessità economica. Perciò l'Economic Complexity Index (ECI) viene definito come:

$$
\mathrm{ECI}=\frac{\vec{K}-<\vec{K}>}{\operatorname{stdev}(\vec{K})}
$$

Dove $<>$ indica la media, stdev indica la deviazione standard e $\vec{K}$ è l'autovettore di $\widehat{M_{c C l}}$ associato al secondo più grande autovalore.

\section{d) Strategia di stima}

La strategia di stima poggia, da un punto di vista teorico, su un modello di crescita semplificato con ritorni di scala costanti e l'impiego di capitale fisico, occupazione e capitale umano tra i fattori della produzione. Lo steady state, utilizzando il modello di Mankiw et al. (1992), è dato da: 


$$
\log P I L_{i t}=\log T e c n_{i}+\beta_{1} \log C F_{i t}+\beta_{2} \log C U_{i t}+\beta_{3} O C C_{i t}
$$

dove $\log P I L$ è il Pil reale pro capite, logTecn è la componente tecnologica, $\log C F$ e $\log C U$ rappresentano la propensione ad accumulare asset fisici e capitale umano, $O C C$ il tasso di crescita dell'occupazione.

L'andamento della componente tecnologica viene espressa con la seguente funzione esponenziale:

$\operatorname{Tecn}_{i t}=\operatorname{Tecn}_{0} e^{\vartheta_{i} t}$

dove $\vartheta_{i}$ è il coefficiente di crescita della componente tecnologica per il paese $i$.

Riteniamo che tale coefficiente sia influenzato sia dalla spesa in ricerca e sviluppo (R\&S) che dal grado di complessità del sistema economico. Quindi,

$\vartheta_{i} t=\alpha_{t}+\alpha_{1} R \& D_{i t}+\alpha_{2} E C I_{i t}$

Inserendo la componente tecnologica modificata (13) nella specificazione diff-in-diff della (12) possiamo scrivere:

$\log P I L_{i t}=\alpha_{i}+\alpha_{t}+\alpha_{1} R \& D_{i t}+\alpha_{2} E C I_{i t}+\beta_{1} \log C F_{i t}+$ $\beta_{2} \log C U_{i t}+\beta_{3} O C C_{i t}+\varepsilon_{i t}$

in cui $\alpha_{i}$ e $\alpha_{t}$ sono rispettivamente l'effetto fisso paese e tempo.

L'analisi parte stimando la (15) con l'uso di diverse tecniche di stima: OLS pooled, panel a effetti fissi e random.

La rilevanza della dinamica associata alla crescita economica, tuttavia, suggerisce che i coefficienti OLS possano essere inconsistenti in virtù della correlazione tra $\log P I L_{i t-1}$ e il termine di errore, anche laddove si utilizzasse un approccio che preveda il ricorso alle differenze prime; tale correlazione determinerebbe, quindi, un problema di endogeneity bias nelle stime.

Per questo motivo, al fine di corroborare la robustezza dei risultati, viene condotta un'analisi panel dinamica (Holtz-Eakin et al., 1988; Arellano e Bond, 1991; Arellano e Bover, 1995; Blundell e Bond, 1998) con il ricorso al modello auto-regressivo system-GMM (Arellano e Bond, 1991; Arellano e Bover, 1995).

Pertanto, la funzione di stima (15), in questo caso, assume la seguente forma: 


$$
\begin{aligned}
& \log P I L_{i t}=\alpha_{i}+\alpha_{t}+\log P I L_{i t-1}+\alpha_{1} R \& D_{i t}+\alpha_{2} E C I_{i t}+ \\
& \beta_{1} \log C F_{i t}+\beta_{2} \log C U_{i t}+\beta_{3} O C C_{i t}+\varepsilon_{i t}
\end{aligned}
$$

dove $\alpha_{i}$ e $\alpha_{t}$ sono rispettivamente gli effetti fissi paese e tempo, $\log P I L_{i t-1}$ è il valore del Pil reale pro capite del periodo precedente. Lo stimatore dinamico system-GMM combina la (16) sia in differenze che in livelli; ovvero, il system-GMM prevede che accanto alle equazioni originali in differenze strumentate con i livelli (approccio difference-GMM) si combinino le equazioni in livelli strumentate con le differenze.

Il metodo di stima system-GMM fornisce una prova di robustezza che consente di controllare per la presenza di: i) endogeneità dei regressori; ii) effetti fissi tra gruppi; iii) eteroschedasticità e auto-correlazione tra gruppi. La validità dell'approccio strumentale GMM è assicurata dal ricorso ai test di correlazione seriale di primo e secondo ordine (Arellano e Bond, 1991) ed al test di Sargan per la sovra-identificazione degli strumenti.

$\mathrm{Al}$ fine di approfondire la relazione funzionale tra $\mathrm{ECI}$ e crescita consideriamo, infine, il seguente modello semi-parametrico a effetti fissi (Baltagi e Li, 2002):

$$
\log P I L_{\mathrm{it}}=\alpha_{\mathrm{i}}+\alpha_{t}+\beta_{X} X_{i t}+\mathrm{g}\left(E C I_{\mathrm{it}}\right)+v_{\mathrm{it}}
$$

in cui ECI entra in modo non lineare nella componente non parametrica. Stimiamo $g(\cdot)$ utilizzando un modello $B$-spline di ordine $k=4$.

\section{Principali risultati}

In tabella 3 vengono esposti i risultati di stima relativi ai modelli pooled OLS, panel ad effetti fissi (P-FE) e panel ad effetti random (P-RE). Le stime sono condotte includendo gli effetti fissi "tempo" e "paese".

Complessivamente appare rilevante notare che, a dispetto delle diverse assunzioni alla base dei modelli di stima utilizzati, i risultati sono stabili, soprattutto per quel che riguarda il segno e la magnitudine del coefficiente della nostra variabile di complessità economica (ECI).

Il risultato dell'Hausman test, tuttavia, rivela che il modello appropriato di riferimento sia quello ad effetti fissi. La null hypothesis che il modello random sia appropriato rispetto all'ipotesi alternativa ad effetti fissi, infatti, è rigettata. 
Tab. 3 - Primi risultati di stima: Pooled OLS, Panel Fixed, Random Effects.

\begin{tabular}{|c|c|c|c|c|c|c|}
\hline Variabile Dip. Log. PIL & $\begin{array}{l}\text { POOLED OLS } \\
\text { (a) }\end{array}$ & $\begin{array}{c}\text { P-FE } \\
\text { (b) }\end{array}$ & $\begin{array}{c}\mathbf{P}-\mathbf{R E} \\
(c)\end{array}$ & $\begin{array}{l}\text { POO- } \\
\text { LED } \\
\text { OLS } \\
(d) \\
\end{array}$ & $\begin{array}{c}\text { P-FE } \\
\text { (e) }\end{array}$ & $\begin{array}{c}\text { P-RE } \\
(f)\end{array}$ \\
\hline $\begin{array}{l}\text { Log. Inv. } \\
\text { fissi lordi }\end{array}$ & $\begin{array}{l}-0.585 * * * \\
(0.152)\end{array}$ & $\begin{array}{l}0.152 * * * \\
(0.028)\end{array}$ & $\begin{array}{l}0.147 * * * \\
(0.028)\end{array}$ & $\begin{array}{l}0.748 * * * \\
(0.153)\end{array}$ & $\begin{array}{l}0.121 * * * \\
(0.029)\end{array}$ & $\begin{array}{l}0.122 * * * \\
(0.031)\end{array}$ \\
\hline Log. Occupazione & $\begin{array}{l}1.624 * * * \\
(0.340)\end{array}$ & $\begin{array}{l}0.911 * * * \\
(0.091)\end{array}$ & $\begin{array}{l}0.933 * * * \\
(0.093)\end{array}$ & $\begin{array}{l}2.129 * * * \\
(0.312)\end{array}$ & $\begin{array}{l}0.634 * * * \\
(0.094)\end{array}$ & $\begin{array}{l}0.690 * * * \\
(0.102)\end{array}$ \\
\hline Istruzione terziaria & $\begin{array}{l}0.779 * * * \\
(0.110)\end{array}$ & $\begin{array}{l}-0.037 \\
(0.041)\end{array}$ & $\begin{array}{l}-0.020 \\
(0.042)\end{array}$ & $\begin{array}{l}0.650 * * * \\
(0.083)\end{array}$ & $\begin{array}{l}0.023 \\
(0.039)\end{array}$ & $\begin{array}{l}0.037 \\
(0.042)\end{array}$ \\
\hline$\Delta$ Pop. in età lavorativa & $\begin{array}{l}0.973 * * * \\
(0.130)\end{array}$ & $\begin{array}{l}-0.023 \\
(0.016) \\
\end{array}$ & $\begin{array}{l}-0.020 \\
(0.017) \\
\end{array}$ & $\begin{array}{l}0.007 \\
(0.014) \\
\end{array}$ & $\begin{array}{l}-0.007^{*} \\
(0.004) \\
\end{array}$ & $\begin{array}{l}-0.009^{*} \\
(0.005) \\
\end{array}$ \\
\hline ECI & $\begin{array}{l}0.628 * * * \\
(0.067)\end{array}$ & $\begin{array}{l}0.276^{* * *} \\
(0.038)\end{array}$ & $\begin{array}{l}0.285^{* * * *} \\
(0.039)\end{array}$ & $\begin{array}{l}0.175^{* *} \\
(0.076)\end{array}$ & $\begin{array}{l}0.335 * * * \\
(0.037)\end{array}$ & $\begin{array}{l}0.346^{* * *} \\
(0.040)\end{array}$ \\
\hline Spesa pubblica in $R \& S$ & & & & $\begin{array}{l}0.875 * * * \\
(0.150)\end{array}$ & $\begin{array}{l}0.164 * * * \\
(0.034)\end{array}$ & $\begin{array}{l}0.138 * * * \\
(0.037)\end{array}$ \\
\hline $\begin{array}{l}\text { Spesa privata } \\
\text { in } R \& S\end{array}$ & & & & $\begin{array}{l}0.111^{*} \\
(0.066) \\
\end{array}$ & $\begin{array}{l}0.039 * * \\
(0.015) \\
\end{array}$ & $\begin{array}{l}0.031^{*} \\
(0.017) \\
\end{array}$ \\
\hline Debito pubblico & & & & $\begin{array}{l}- \\
0.011 * * * \\
(0.001)\end{array}$ & $\begin{array}{l}-0.001 * * * \\
(0.000)\end{array}$ & $\begin{array}{l}-0.001 * * * \\
(0.000)\end{array}$ \\
\hline Tempo & Yes & Yes & Yes & Yes & Yes & Yes \\
\hline Cost. & $\begin{array}{l}1.693 \\
(1.270)\end{array}$ & $\begin{array}{l}5.470 * * * \\
(0.394)\end{array}$ & $\begin{array}{l}5.329^{* * * *} \\
(0.412)\end{array}$ & $\begin{array}{l}5.357 * * * \\
(1.773)\end{array}$ & $\begin{array}{l}7.047 * * * \\
(0.517)\end{array}$ & $\begin{array}{l}6.838 * * * \\
(0.567)\end{array}$ \\
\hline $\begin{array}{l}\text { F-stat./Wald } \chi^{2} \\
\mathrm{R}^{2}\end{array}$ & $\begin{array}{l}26.59 * * * \\
0.59\end{array}$ & $\begin{array}{l}69.89^{* * * *} \\
0.81\end{array}$ & $\begin{array}{l}998.77 * * * \\
0.80\end{array}$ & $\begin{array}{l}46.71 \text { *** } \\
0.75\end{array}$ & $\begin{array}{l}59.27 * * * \\
0.81\end{array}$ & $\begin{array}{l}954.89^{* * *} \\
0.81\end{array}$ \\
\hline $\begin{array}{l}\text { Gruppi } \\
\text { Osservazioni }\end{array}$ & $\begin{array}{l}27 \\
295\end{array}$ & $\begin{array}{l}27 \\
295\end{array}$ & $\begin{array}{l}27 \\
295\end{array}$ & $\begin{array}{l}27 \\
295\end{array}$ & $\begin{array}{l}27 \\
295\end{array}$ & $\begin{array}{l}27 \\
295\end{array}$ \\
\hline
\end{tabular}

Pertanto, volgendo l'attenzione alla stima del modello (b) è possibile osservare quanto segue. La stima panel a effetti fissi su 27 paesi e con 295 osservazioni supporta l'ipotesi alla base del presente lavoro. La complessità dei sistemi economici, infatti, rivelando la quantità di conoscenza che è incorporata nella struttura produttiva di un'economia, rappresenta una determinante molto rilevante del Pil reale pro capite. Il coefficiente dell'ECI, infatti, è positivo e altamente significativo (1\%). In altre parole, anche controllando per tutte le variabili del modello di crescita endogena (Mankiw et al., 1992), l'indicatore di complessità economica ECI, incluso nella componente tecnologica, conferma la sua qualità di determinante del Pil reale pro capite. Tra le altre variabili del modello, inoltre, appare interessante notare che, in linea con le ipotesi tradizionali, sia gli investimenti fissi lordi (0.152), sia l'occupazione (0.911), presentano segni positivi e risultano altamente significativi (1\%). Questo risultato conferma che per il nostro panel, ceteris paribus, il Pil reale pro capite cresce al crescere degli asset fisici e del capitale umano.

Al fine di verificare l'ipotesi che la spesa in Ricerca e Sviluppo non possa da sola cogliere tutti i fattori rivelatori del grado di complessità dei sistemi innovativi, nei modelli da $(d)$ a $(f)$, includendo nella specificazione 
la spesa privata e pubblica in $\mathrm{R} \& \mathrm{~S}$, controlliamo se la relazione tra ECI e Pil resti influente e statisticamente robusta. Specificando la componente tecnologica con la eq. (14), l'ECI continua a presentare un segno positivo, una simile magnitudine del coefficiente e una alta significatività statistica $(1 \%)$.

Quindi, tale risultato conferma che il grado di complessità dei sistemi economici rappresenta una variabile chiave per cogliere l'eterogeneità tra $\mathrm{i}$ paesi dei diversi trend di crescita del Pil reale osservati.

Come da attese, sia la spesa in R\&S pubblica che quella privata presentano il segno positivo e sono statisticamente significative al $1 \%$ e al $10 \%$ rispettivamente. Di rilievo considerare che la spesa in $\mathrm{R} \& \mathrm{~S}$ pubblica presenta un coefficiente significativamente più grande del coefficiente della spesa privata; ceteris paribus, la spesa pubblica in R\&S mostra un effetto moltiplicatore di 4.5 volte circa più grande di quello determinato dalla spesa privata. Al fine di abilitare un confronto tra paesi tenendo conto della diversa resilienza dei conti pubblici, inseriamo tra i controlli il Debito Pubblico.

Al fine di approfondire il legame funzionale tra ECI e crescita stimiamo l'eq. (17) con lo stimatore semi-parametrico ad effetti fissi (Baltagi e Li, 2002), ovvero senza imporre una specifica forma funzionale nella stima di tale relazione. L'ECI, infatti, entra nel modello nella componente non parametrica, mentre nella parte parametrica vengono inserite tutte le altre determinanti classiche della crescita. La stima della relazione tra ECI e Pil reale (metodo $B$-spline di ordine $k=4$ ) è riportata nella sottostante figura 1 .

Tale risultato, oltre a fornire una prova di robustezza rispetto ai risultati ottenuti con i metodi precedenti, mette in luce che al crescere del grado di economic complexity il Pil associato cresce a tassi decrescenti. In altre parole, l'eterogeneità osservata delle performance di crescita dei diversi paesi, al margine, è in larga misura imputabile al diverso grado di complessità economica, specie quando confrontiamo, nel panel, paesi con Pil reale pro capite relativamente più basso. La rilevanza della dinamica associata alla crescita economica, tuttavia, suggerisce che i coefficienti OLS possano essere inconsistenti in virtù della correlazione tra $\log P I L_{i t-1}$ e il termine di errore, anche laddove si utilizzasse un approccio che preveda il ricorso alle differenze prime; tale correlazione determinerebbe, quindi, un problema di endogeneity bias nelle stime. Per tale ragione al fine di verificare la robustezza delle stime precedenti, stimiamo l'eq. (16) utilizzando il system-GMM. 
Figura 1 - Modello Semi-parametrico ad effetti fissi

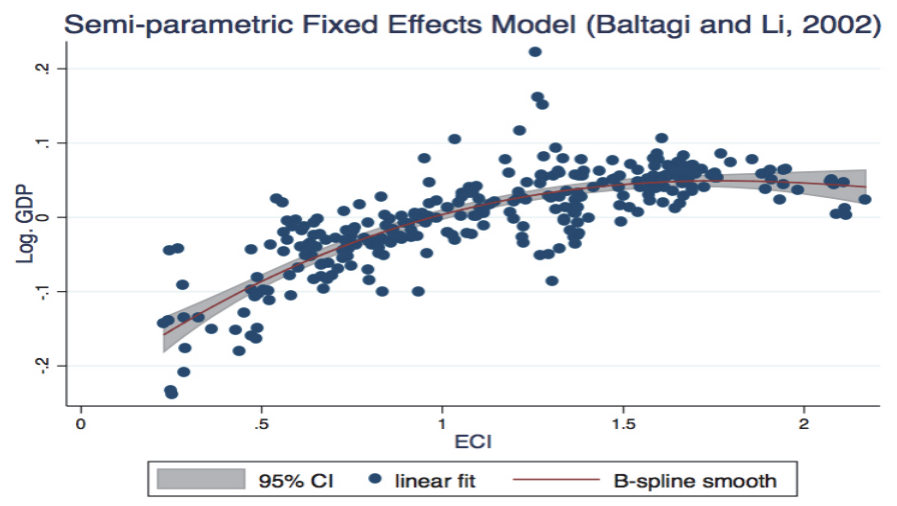

In tabella 4 riportiamo i risultati di stima considerando le medesime specificazioni del modello utilizzate in precedenza (ossia senza e con l'inclusione della spesa pubblica e privata in R\&S).

I risultati ottenuti attraverso questa ulteriore tecnica di stima confermano definitivamente i findings emersi nelle stime precedenti. L'indicatore di complessità economica (ECI), anche nella stima panel auto-regressiva dinamica, oltre a conservare il segno e la significatività statistica ottenuti nelle stime precedenti, presenta una magnitudine del coefficiente che è in linea con i valori riportati in tabella 3 .

Tab. 4 - Secondi risultati di stima: GMM

$\begin{array}{lcc} & \text { GMM } & \text { GMM } \\ \text { Variabile Dip. Log. PIL } & (a) & (b)\end{array}$

\begin{tabular}{lll}
\hline Log. PIL $t-1$ & $0.385^{* * *}$ & $0.320^{* * *}$ \\
& $(0.033)$ & $(0.036)$ \\
Log. Investimenti fissi lordi & 0.017 & 0.006 \\
& $(0.015)$ & $(0.017)$ \\
Log. Occupazione & $0.619 * * *$ & $0.501 * * *$ \\
& $(0.067)$ & $(0.067)$ \\
Istruzione terziaria & -0.016 & -0.014 \\
& $(0.036)$ & $(0.036)$ \\
$\Delta$ Popolazione in età lavorativa & -0.005 & $-0.011^{* * *}$ \\
& $(0.003)$ & $(0.003)$ \\
ECI & $0.193 * * *$ & $0.211^{* * *}$ \\
& $(0.025)$ & $(0.024)$
\end{tabular}




\begin{tabular}{lll} 
Spesa pubblica in R\&S & & $0.062^{* *}$ \\
& & $(0.025)$ \\
Spesa privata in R\&S & & 0.006 \\
& & $(0.011)$ \\
Debito pubblico & & $-0.001^{* * *}$ \\
Tempo & Yes & $(0.000)$ \\
Cost. & $3.603^{* * *}$ & Yes \\
& $(0.305)$ & $5.317^{* * *}$ \\
Wald $\chi^{2}$ & $9941.79^{* * *}$ & $(0.398)$ \\
AR $(1) p$-value & 0.11 & $22259.87 * * *$ \\
AR $(2) p$-value & 0.43 & 0.47 \\
Sargan & 8.84 & 0.61 \\
\hline Gruppi & 27 & 11.04 \\
Oss. & 295 & 27 \\
\hline Note: Robust standard errors in parentheses $* * *$ & $\mathrm{p}<0.01,{ }^{* *} \mathrm{p}<0.05, * \mathrm{p}<0.1$
\end{tabular}

\section{Conclusioni}

I modelli tradizionali di crescita, pur dando rilevanza alle componenti endogene dello sviluppo economico, non sembrano considerare l'impatto generato dalle interazioni complesse dei diversi fattori della crescita. In tale prospettiva, il presente lavoro è rivolto a costruire un ponte tra la recente letteratura sulla complessità economica e i modelli teorici della crescita endogena.

La gamma dei beni che un paese può produrre, infatti, rappresenta una misurazione indiretta delle capabilities e del posizionamento competitivo dei diversi sistemi economici, ovvero, fornisce una misura della complessità del suo tessuto economico e produttivo, altrimenti difficilmente osservabile tramite l'inclusione di variabili di tipo tradizionale.

Con un approccio empirico, il nostro lavoro ha testato se l'inclusione all'interno dei modelli di crescita endogena di uno specifico indicatore di complessità economica - ossia l'ECI (Hidalgo e Hausmann, 2009; Hausmann et al., 2011, Hausmann et al., 2013; Hidalgo, 2015) - contribuisca a migliorare l'analisi della dinamica di crescita dell'economia.

Attraverso un'analisi empirica panel (27 paesi, 2007-2018) di tipo tradizionale (pooled OLS, panel a effetti fissi e random) e dinamica (systemGMM) troviamo conferma che il grado di complessità dei sistemi economici rappresenta una variabile chiave per cogliere l'eterogeneità dei trend di cre- 
scita osservati nei diversi paesi del panel. Al crescere del grado di complessità economica, ceteris paribus, si associano livelli di Pil reale pro capite più elevati. L'approccio semi-parametrico ad effetti fissi, infine, rivela che la relazione che lega l'ECI al Pil è non lineare.

Vista la capacità del grado di complessità dei sistemi economici, come definita da Hausmann et al. (2011), di cogliere il trend di crescita dei diversi paesi del nostro panel, per i policy maker è possibile trarre una implicazione di politica industriale ed economica. Nell' ambito della scelta del mix di politiche pubbliche da utilizzare per promuovere la crescita economica, appare fondamentale poggiarsi sulla complementarietà tra le politiche selettive di sostegno alla ricerca, sviluppo e innovazione (R\&S\&I) e le politiche di sostegno alle componenti strutturali dell'ecosistema innovativo nel suo insieme. Tale approccio richiede che, unitamente agli interventi selettivi a sostegno delle diverse traiettorie tecnologiche ritenute prioritarie nell'ambito della strategia di specializzazione intelligente (SSS), siano attivate misure di intervento orizzontale volte a ridurre le frizioni (market and administrative burdens) presenti nel mercato dell'innovazione italiano. Tali strumenti eterogenei e multiformi sono volti a innalzare l'efficienza statica e dinamica dell'ecosistema innovativo nazionale attraverso il sostegno alle infrastrutture di ricerca fisiche e non (acceleratori e incubatori d'impresa, centri di competenza, infrastrutture intelligenti, internet of things, ecc.), il sostegno alla creazione di reti di ricerca industriale internazionali, il ricorso a interventi strutturali in bilancio di matrice fiscale per sostenere gli investimenti innovativi nel medio-lungo periodo.

\section{Riferimenti bibliografici}

Aghion P., Howitt P. (1992). A model of growth through creative destruction. Econometrica, 60: 323-351. DOI: 10.2307/2951599

Aghion P., Howitt P. (1998). Endogenous growth theory. MIT Press.

Arellano M., Bond S. (1991). Some tests of specification for panel data: Monte Carlo evidence and an application to employment equations. The Review of economic studies, 58(2): 277-297. DOI: 10.2307/2297968

Arellano M., Bover O. (1995). Another look at the instrumental-variable estimation of error-components models. Journal of econometrics, 68: 29-51. DOI: 10.1016/0304-4076(94)01642-d

Arrow K. (1962). The economic implications of learning by doing. The Review of economic studies, 29: 155-73. DOI: $10.2307 / 2295952$

Arthur W.B. (1999). Complexity and the economy. Science, 284: 107-109. DOI: 10.1126/science.284.5411.107

Balassa B. (1965), Trade Liberalisation and Revealed Comparative Advantage. The Manchester school, 33: 99-123. DOI: 10.1111/j.1467-9957.1965.tb00050.x 
Baltagi B.H., Li D. (2002). Series estimation of partially linear panel data models with fixed effects. Annals of Economics and Finance, 3(1): 103-116.

Barro R. (1990). Government spending in a simple model of endogenous growth. Journal of political economy, 98: S103-S105. DOI: 10.1086/261726

Becker G.S. (1964). Human capital: a theoretical and empirical analysis, with special reference to education. New York: National Bureau of Economic Research: Distributed by Columbia University Press.

Beinhocker E.D. (2006). The origin of wealth: Evolution, complexity, and the radical remaking of economics. Harvard Business Press.

Blundell R., Bond S. (1998). Initial conditions and moment restrictions in dynamic panel data models. Journal of Econometrics, 87: 115-143. DOI: 10.1016/s03044076(98)00009-8

Chenery H.B. (1960). Patterns of industrial growth. The American Economic Review, 50(4): 624-654. https://www.jstor.org/stable/1812463

Domar E. (1946). Capital expansion, rate of growth and employment. Econometrica, 14: 137-147. DOI: $10.2307 / 1905364$

Ferrarini B., Scaramozzino P. (2016). Production complexity, adaptability and economic growth. Structural change and economic dynamics, 37: 52-61. DOI: 10.1016/j.strueco.2015.12.001

Fukuyama F. (1996). Trust: Human nature and the reconstitution of social order. Free Press Paperbacks.

Furtado C. (1964). Development and underdevelopment. University of California Press.

Gala P., Rocha I., Magacho G. (2018). The structuralist revenge: economic complexity as an important dimension to evaluate growth and development. Brazilian journal of political economy, 38: 219-236. DOI: 10.1590/0101-31572018v38n02a01

Grossman G., Helpman E. (1991). Quality ladders in the theory of growth. The Review of economic studies, 58: 43-61. DOI: 10.2307/2298044

Harrod R.F. (1939). An essay in dynamic theory. The economic journal, 49: 14-33. DOI: $10.2307 / 2225181$

Hausmann R., Hidalgo C.A., Bustos S., Coscia M., Chung S., Jimenez J., Simoes A., Yildirim M.A. (2011). The atlas of economic complexity. Mapping paths to prosperity. Growth Lab at Harvard University.

Hausmann R., Hidalgo C.A., Bustos S., Coscia M., Simoes A., Yildirim M.A. (2013). The atlas of economic complexity: Mapping paths to prosperity. MIT Press.

Hausmann R., Klinger B. (2007). The structure of the product space and the evolution of comparative. Center for International Development at Harvard University, Working Paper No.146.

Hidalgo C.A. (2015). Why information grows: The evolution of order, from atoms to economies. New York: Penguin Press.

Hidalgo C.A., Hausmann R. (2009). The building blocks of economic complexity. PNAS 106: 10570-10575. DOI: 10.1073/pnas.0900943106

Hirschman A.O. (1958). The strategy of economic development. New Haven: Yale University Press.

Holtz-Eakin D., Newey W., Rosen H.S. (1988). Estimating vector autoregressions with panel data. Econometrica, 56: 1371-1395. DOI: 10.2307/1913103 
Kaldor N. (1967). Strategic factors in economic development. New York State School of industrial and labor relations. Ithaca, NY.

Kuznets S. (1966). Modern economic growth: rate, structure and spread. Yale University Press. New Haven, CT.

Lewis A.W. (1954). Economic development with unlimited supplies of labour. The Manchester school, 22: 139-191. DOI: 1467-9957.1954.tb00021.x

Lucas R.E. (1988). On the mechanics of economic development. Journal of monetary economics, 22: 3-42. DOI: 10.1016/0304-3932(88)90168-7

Mankiw N.G., Romer D., Weil D.N. (1992). A contribution to the empirics of economic growth. Quarterly journal of economics, 107: 407-437. DOI: 10.2307/2118477

Mitchell M. (2009). Complexity. A guided tour. Oxford University Press.

Myrdal G. (1957). Economic theory and underdeveloped regions. London: Gerald Duckworth.

Nurkse R. (1953). The problem of capital formation in underdeveloped countries. Oxford: Basil Blackwell.

Prebisch R. (1950). The economic development of Latin America and its principal problems. United Nations, New York.

Prebisch R. (1959). Commercial policy in underdeveloped countries. The American Economic Review, 49(2): 251-73. https://www.jstor.org/stable/1816120

Romer P.M. (1986). Increasing returns and long-run growth. Journal of political economy, 94: 1002-1037. DOI: 10.1086/261420

Romer P.M. (1990). Endogenous technological change. Journal of political economy, 98: S71-S102. DOI: 10.1086/261725

Romer P.M. (1994). The origins of endogenous growth. The Journal of economic perspectives, 8: 3-22. DOI: 10.1257/jep.8.1.3

Rosenstein-Rodan P.N. (1943). Problems of industrialisation of eastern and southeastern Europe. The economic journal, 53: 202-211. DOI: 10.2307/2226317

Rostow W.W. (1959). The stages of economic growth. The economic history review, 12: 1-16. DOI: 10.1111/j.1468-0289.1959.tb01829.x

Sbardella A., Pugliese E., Zaccaria A., Scaramozzino P. (2018). The role of complex analysis in modelling economic growth. Entropy, 20: 883. DOI: 10.3390/e20110883

Schultz T.W. (1963). The economic value of education. New York: Columbia University Press.

Singer H.W. (1950). The distribution of gains between investing and borrowing countries. The American Economic Review, 40(2): 473-485. WOS: 000203281600038

Solow R.M. (1956). A contribution to the theory of economic growth. The quarterly journal of economics, 70: 65-94. DOI: 10.2307/1884513

Stojkoski V., Kocarev L. (2017). The relationship between growth and economic complexity: Evidence from southeastern and central Europe. MPRA Paper No. 77837 